\title{
Facilitated vascular interrupted anastomosis in cardiovascular surgery with a new clip device
}

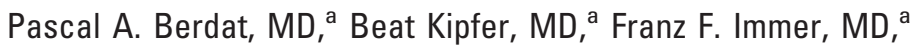 \\ Jean-Pierre Pfammatter, MD, ${ }^{\mathrm{b}}$ and Thierry Carrel, MD, ${ }^{\mathrm{a}}$ Bern, Switzerland
}

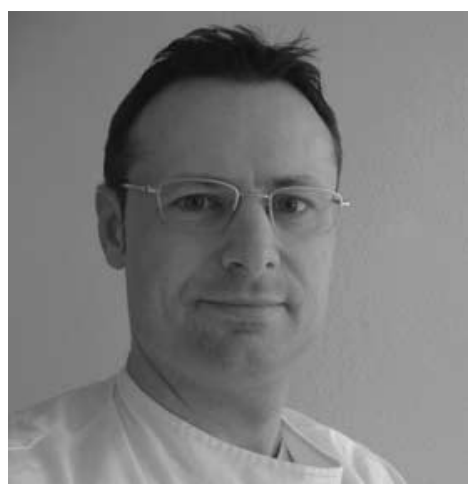

Pascal A. Berdat

1 lthough confection of hand-sewn vascular anastomoses with interrupted sutures demands considerable skill and can be very time consuming, it has the advantage of avoiding a potential purse-string stricture on the anastomosis, allows for a greater anastomotic orifice, has better pulsatility of the anastomosis, and has unrestricted growth potential in comparison with running suture techniques. ${ }^{1-3}$ Consequently, short- and long-term patency and clinical outcome might be improved by reducing anastomotic stricture, thrombosis, and occlusion. Interrupted sutures might therefore be preferable in children because of the growth of vascular structures and in cases in which low-pressure vessels are involved. ${ }^{4}$ However, because of the time-consuming process, which might affect immediate outcome by prolonging cardiopulmonary bypass and myocardial ischemia, requiring considerable manipulation of the vessels, and requiring extensive knot tying for confection of such anastomoses, many surgeons prefer to use running sutures. Coalescent Surgical, Inc (Sunnyvale, Calif) has developed a nitinol self-coiling penetrating clip, the U-clip, to facilitate confection of vascular anastomoses in an interrupted fashion. The device consists of a vascular needle on a nitinol filament of 3 or $6 \mathrm{~cm}$ with a nitinol clip mounted on a release mechanism at the end (Figure 1). When the release mechanism is squeezed, the clip is deployed, self-recoiling, closing, and eliminating knot tying and assisted suture management (Figure 2). Five different sizes of U-clips exist to fit a broad spectrum of vessels.

The U-clips have been tested in a bovine model of a right internal thoracic artery to coronary anastomosis ${ }^{5}$ and compared

\footnotetext{
From the Department of Cardiovascular Surgery ${ }^{\mathrm{a}}$ and Division of Pediatric Cardiology, ${ }^{\text {b }}$ University Hospital, Bern, Switzerland.

Received for publication Nov 21, 2001; accepted for publication April 23, 2002.

Address for reprints: P. A. Berdat, MD, Clinic for Cardiovascular Surgery, Swiss Cardiovascular Center Bern, Inselspital, CH-3010 Bern, Switzerland (E-mail: pascal.berdat@insel.ch).

J Thorac Cardiovasc Surg 2002;124:1256-8

Copyright (C) 2002 by The American Association for Thoracic Surgery $0022-5223 / 2002 \$ 35.00+0 \quad \mathbf{1 2 / 5 4 / 1 2 6 8 0 8}$

doi: $10.1067 / \mathrm{mtc} .2002 .126808$
}

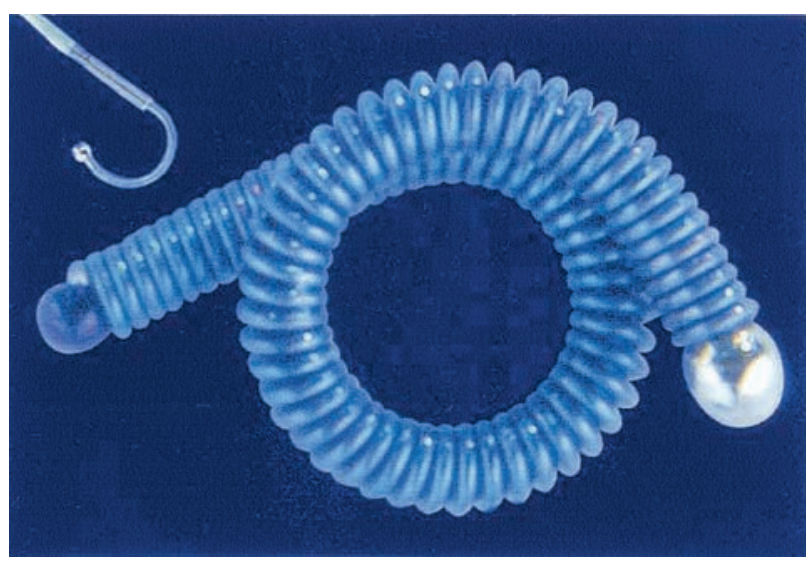

Figure 1. View of the U-clip, a self-recoiling nitinol clip mounted on a flexible member with a needle (inset: before release).

with polypropylene sutures in a carotid arteriotomy closure model. Intraoperative, 1-week, 8-week, and 26-week angiograms and histopathologic examinations showed excellent graft patency and healing characteristics. By October 2000, CE marking was obtained, and the device was introduced to the European market. We report the first successful clinical use of the U-clip in congenital cardiac surgery and our initial clinical experience with this device in cardiac and peripheral vascular procedures.

\section{Patients and Methods}

U-clips of different sizes were used for confection of various vascular anastomoses in 11 patients with a mean age of $46.2 \pm$ 31.8 years (range, 4 days to 76.2 years).

The U-clips were applied as described earlier (Figure 2). Care was taken to evenly distribute the U-clips around the anastomotic edges to create an optimal anastomotic geometry and local hemostasis.

\section{Results}

U-clips were used in $82 \%$ of all vascular anastomoses made (Figure 3). In 5 patients the U-clips were used for confection of 12 


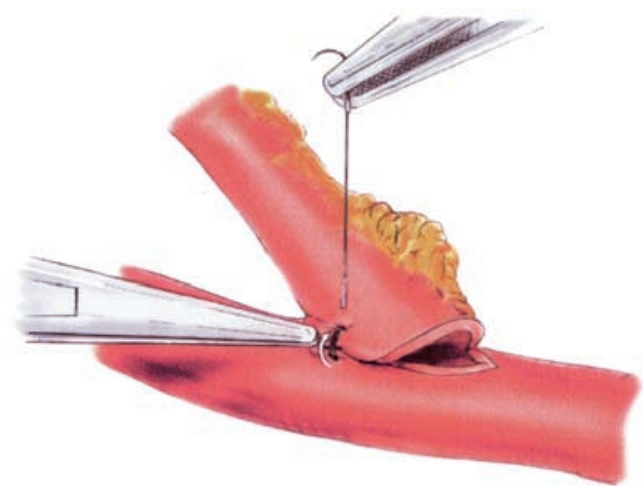

A

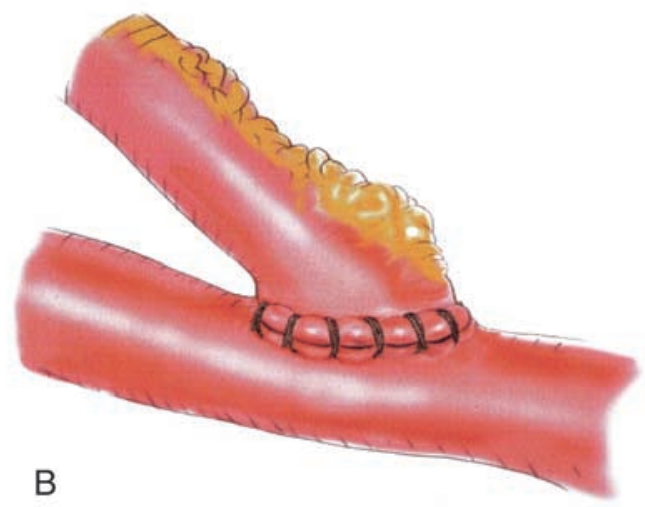

B

Figure 2. Schematic view of end-to-side anastomosis with the use of U-clips. A, The needle has been passed through both vessel walls with the U-clip positioned and deployed. B, The completed anastomosis.
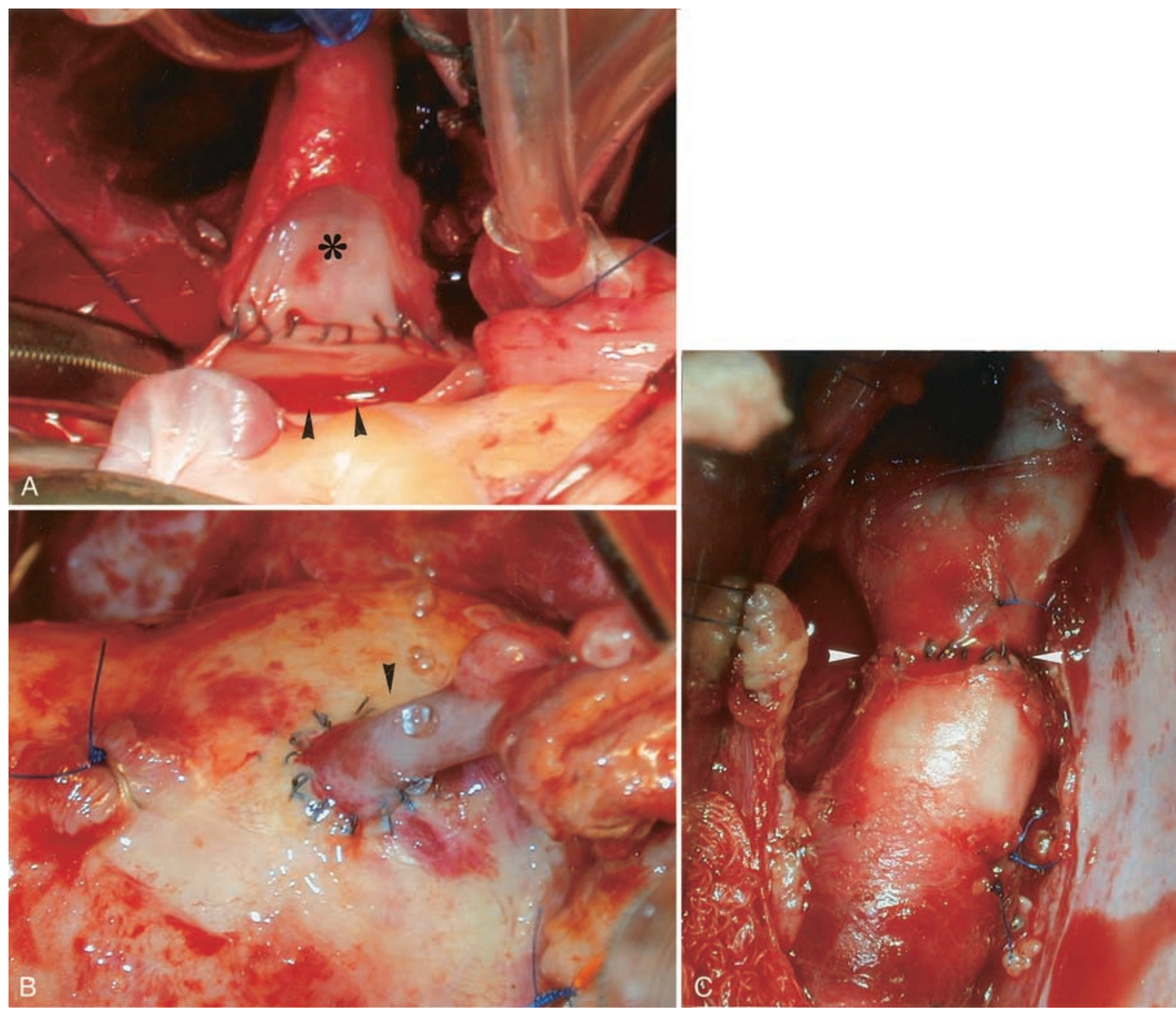

Figure 3. Intraoperative views. A, Bidirectional Glenn anastomosis (asterisk, superior vena cava; black arrows, right pulmonary artery) during confection: the dorsal suture line has already been constructed. B, Proximal anastomosis of the radial artery coronary bypass graft (black arrow) on the ascending aorta is shown. C, End-to-end anatomosis (white arrows) of the proximal descending aorta after resection of a coarctation is shown. 
coronary bypass graft anastomoses with saphenous vein grafts, radial arteries, and internal thoracic arteries. U-clips of size 50 were used proximally, and U-clips of sizes 18 and 20 were used distally.

U-clips were used for the first time in 3 pediatric patients for a bidirectional Glenn anastomosis in a 28-month-old infant (size 50), for a modified Blalock-Taussig shunt with a 4-mm expanded polytetrafluoroethylene shunt (IMPRA, Inc, Tempe, Ariz) in a newborn with pulmonary atresia with intact ventricular septum (size 18), and for coarctation repair in another 4-year-old boy (size 50).

In 3 adults reconstruction of peripheral vessels was performed with U-clips. In one patient reconstruction of the popliteal artery was performed with end-to-end anastomosis (size 50). In the second patient reconstruction of the external iliac artery after cannulation for cardiopulmonary bypass was performed (size 50). In another patient with chronic renal failure, confection of a Cimino-Breshia arteriovenous fistula on the forearm was performed (size 18).

In all cases the use of the U-clips was easy, and no technical difficulties were encountered. Immediate anastomotic hemostasis was excellent, even with the use of foreign material. No special surgical instrument was needed for confection of anastomoses with the U-clip. Although we did not measure the precise time for construction of such anastomoses, this technique seemed much faster than conventional interrupted sutures and as fast as continuous sutures. Excellent flow was demonstrated in all cases by means of transit-time Doppler scanning or invasive pressure gradient measurements and confirmed by means of postoperative echocardiography when appropriate. Short-term outcome with a mean follow-up of $6 \pm 3.8$ months was uneventful in all but the newborn with pulmonary atresia with intact ventricular septum, who died suddenly after 62 days.

\section{Comment}

The new U-clips allow easy, precise, and time-sparing confection of vascular anastomoses in an interrupted fashion in the whole field of cardiovascular surgery. Its application might prevent anastomotic stricture, thrombosis, and occlusion caused by purse-string effects or growth restriction in the pediatric cardiac group by allowing for greater anastomotic diameter, pulsatility of the anastomosis, and unrestricted growth potential in comparison with running sutures. Consequently, long-term patency of bypass grafts and vascular anastomoses, as well as clinical outcome after cardiovascular surgical procedures, will be improved.

\section{References}

1. Tozzi P, Hayoz D, Ruchat P, Corno A, Oedman C, Botta U, et al. Animal model to compare the effects of suture technique on crosssectional compliance on end-to-side anastomoses. Eur J Cardiothorac Surg. 2001;19:477-81.

2. Chiu IS, Hung CR, Chao SF, Huang SH, How SW. Growth of the aortic anastomosis in pigs. Comparison of continuous absorbable suture with nonabsorbable suture. J Thorac Cardiovasc Surg. 1988; 95:112-8.

3. Gillinow AM, Lee AW, Redmond JM, Zehr KJ, Jackson L, Davis EA, et al. Absorbable suture improves growth of venous anastomoses. $J$ Vasc Surg. 1992;16:769-73.

4. Torsello G, Schwartz A, Aulich A, Sandmann W. Absorbable polydioxanone suture for venous anastomoses: experimental studies using venography and transluminal angioscopy. Eur J Vasc Surg. 1987;1: 319-25.

5. Hill AC, Maroney TP, Virmani R. Facilitated coronary anastomosis using a nitinol U-clip device: bovine model. $J$ Thorac Cardiovasc Surg. 2001;121:859-70. 